Research Article

\title{
Stored Coulomb Self-Energy of a Uniformly Charged Rectangular Plate
}

\author{
Orion Ciftja \\ Department of Physics, Prairie View A\&M University, Prairie View, TX 77446, USA \\ Correspondence should be addressed to Orion Ciftja; ogciftja@pvamu.edu
}

Received 21 July 2016; Revised 8 November 2016; Accepted 15 November 2016

Academic Editor: Antonio Scarfone

Copyright (C) 2016 Orion Ciftja. This is an open access article distributed under the Creative Commons Attribution License, which permits unrestricted use, distribution, and reproduction in any medium, provided the original work is properly cited.

\begin{abstract}
A large number of electronic devices contain charged, flat plates (electrodes) as their components. The approximation of considering such components as infinitely large plates is not satisfactory for the current status of consumer electronics where size is now extremely small. In particular, the nanotechnology revolution has made the fabrication of truly finite systems with arbitrary shape and characteristic lengths that measure in nanometers possible. As a result the only accurate approach for such situations is to consider the system realistically as one with a finite size extent. In this work we calculate the amount of electrostatic energy that is stored in a charged finite size electrode that is modelled as a uniformly charged rectangular plate with arbitrary length and width. Nontrivial mathematical transformations allow us to derive a closed form exact expression for the Coulomb self-energy of such a system as a function of its length and width (therefore, shape, too). The exact result derived can be useful to understand the storage process of electrostatic energy as a function of size/shape in uniformly charged plate systems. The result also applies to calculations that deal with the properties of a finite two-dimensional electron gas within the jellium model where the finite jellium domain can have an arbitrary rectangular shape.
\end{abstract}

\section{Introduction}

Many electronic devices have charged, flat plates (squares, rectangles, disks, etc.) as their components. These charged plates (often called electrodes) serve the purpose of steering the current along the proper paths in a device. Although any real electrode is finite in extent, one can often get a good understanding of the system by modelling it as an infinite uniformly charged plate. This simple treatment is very reasonable as long as the distance of interest to the electrode is small in comparison to the characteristic length of the electrode itself. For such a situation it is legitimate to treat the edges as if they were infinitely far away from the point of interest.

However, great advances in nanotechnology have now made the fabrication of very small truly finite systems with arbitrary shape, size, and dimensionality possible [1-8] where treatments that discard the finite size are not accurate. In particular, charged nanoplates are key components of any given nanoelectronic device. Often, their properties are size/shape-dependent and dominated mainly by electrostatic interactions [9-15]. For instance, $\mathrm{LiMnPO}_{4}$ nanoplates have been recently grown experimentally for battery cathode applications [16]. Transmission electron microscope measurements have indicated that such nanoplates are very thin rectangles measuring about 50 nanometers (which is approximately a thousand times thinner than a human hair) and up to 2000 nanometers on a side [16]. For these values, they can be safely approximated as almost ideal two-dimensional (2D) rectangular plates. When charged, such nanoplates can theoretically store some of the highest amounts of electrostatic energy of the rechargeable batteries. The amount of electricity that the material can store can be measured by charging and discharging the nanoplate fast or slowly. In this particular case it was found that when the nanoplates were charged slowly over a day and then discharged just as slowly, the amount of electricity held was extremely large [16]. Improvements on the material (charging and discharging in an hour without substantial reductions of the best performance observed for slow charging and discharging) may lead to important advances in consumer electronics. 
Developments in the field of nanotechnology have also made the fabrication of finite systems of electrons confined in a finite $2 \mathrm{D}$ domain possible [17-22]. The simplest model for a finite two-dimensional electron gas (2DEG) involves the "jellium" approximation where one assumes that a neutralizing positive charge is uniformly spread over a finite $2 \mathrm{D}$ region. While the shape of the jellium background is irrelevant when it comes to bulk properties of a 2DEG in the thermodynamic limit [23-25], this is not the case for finite systems of few electrons confined in a finite region. Finite systems of electrons are sensitive to the geometry of the finite jellium domain (the electron-depleted region) where electrons are trapped. As a result, the properties of a finite 2DEG reflect the shape of the chosen positive finite jellium background $[26,27]$. Even though the assumption of a finite square domain is perfectly legitimate, a rectangular shape of the finite jellium background is a more general choice that applies to a finite 2DEG model.

In this work, we consider a uniformly charged finite rectangular domain (which would represent either a uniformly charged nanoplate or a finite rectangular jellium background for a 2DEG) and calculate exactly the amount of Coulomb electrostatic self-energy stored in that system. We obtain an exact analytic expression for such a quantity as a function of length/width and, thus, shape of the rectangular plate. This way one can show precisely how size and shape influence the electrostatic energy stored in a finite plate with rectangular geometry.

The article is organized as follows. In Section 2 we explain the theory and show the main results. In Section 3 we discuss the key findings and present some concluding remarks. Details of some challenging calculations that are deemed of mathematical interest are provided in Appendices A, B, C, D, $\mathrm{E}$, and $\mathrm{F}$.

\section{Calculation of the Coulomb Self-Energy of a Uniformly Charged Rectangular Plate}

We consider an arbitrarily shaped rectangular plate with length, $L_{x}$, and width, $L_{y}$. We assume that the rectangular plate is uniformly charged and contains a total amount of charge, $Q$. Thus, the uniform surface charge density of the rectangular plate is

$$
\sigma=\frac{Q}{L_{x} L_{y}} .
$$

The Cartesian system of coordinates is chosen so that the rectangular plate is assumed to lie in $x-y$ plane. The origin of the system of coordinates is at the center of the rectangular plate. The $x$-and $y$-axes are chosen to be parallel to plate's edges as shown in Figure 1. For this choice of the coordinative system, the rectangular domain uniformly filled with charge is

$$
\begin{aligned}
D: & -\frac{L_{x}}{2} \leq x \leq+\frac{L_{x}}{2}, \\
& -\frac{L_{y}}{2} \leq y \leq+\frac{L_{y}}{2} .
\end{aligned}
$$

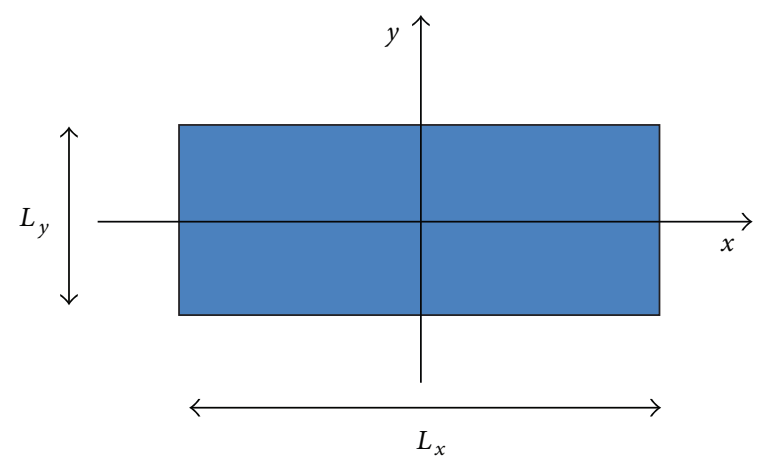

FIGURE 1: Schematic view of a uniformly charged rectangular plate with arbitrary length, $L_{x}$, and width, $L_{y}$. The rectangular plate has a uniform surface charge density, $\sigma=Q /\left(L_{x} L_{y}\right)$, where $Q$ represents the total amount of charge uniformly spread over the area, $L_{x} L_{y}$.

We assume Coulomb interaction between elementary charges $d q_{1}=\sigma d^{2} r_{1}$ and $d q_{2}=\sigma d^{2} r_{2}$, where $\vec{r}_{i}=\left(x_{i}, y_{i}\right)(i=1,2)$ are $2 \mathrm{D}$ position vectors and $d^{2} r_{i}=d x_{i} d y_{i}(i=1,2)$ are elementary surface areas. It is expected that the Coulomb self-energy of the uniformly charged rectangular plate will be shape-dependent. For this reason we denote it as $U\left(L_{x}, L_{y}\right)$ since it will depend on both $L_{x}$ and $L_{y}$. The quantity to calculate is

$$
U\left(L_{x}, L_{y}\right)=\frac{k_{e} \sigma^{2}}{2} \int_{D} d^{2} r_{1} \int_{D} d^{2} r_{2} \frac{1}{\left|\overrightarrow{r_{1}}-\overrightarrow{r_{2}}\right|},
$$

where $k_{e}$ is Coulomb's electric constant and $D$ is the domain of integration as specified in (2). The exact calculation of $U\left(L_{x}, L_{y}\right)$ is very challenging since it involves a nontrivial four-dimensional integral.

In order to simplify the calculation of the integral expression in (3), we transform the quantity $1 /\left|\overrightarrow{r_{1}}-\overrightarrow{r_{2}}\right|$ the following way:

$$
\begin{aligned}
\frac{1}{\left|\overrightarrow{r_{1}}-\overrightarrow{r_{2}}\right|} & =\frac{2}{\sqrt{\pi}} \int_{0}^{\infty} d u e^{-u^{2}\left(\vec{r}_{1}-\vec{r}_{2}\right)^{2}} \\
& =\frac{2}{\sqrt{\pi}} \int_{0}^{\infty} d u e^{-u^{2}\left(x_{1}-x_{2}\right)^{2}} e^{-u^{2}\left(y_{1}-y_{2}\right)^{2}} .
\end{aligned}
$$

Substitution of the result from (4) into (3) leads to

$$
\begin{aligned}
U\left(L_{x}, L_{y}\right)= & \frac{k_{e} \sigma^{2}}{\sqrt{\pi}} \int_{0}^{\infty} d u \int_{-L_{x} / 2}^{+L_{x} / 2} d x_{1} \int_{-L_{x} / 2}^{+L_{x} / 2} d x_{2} \\
& \cdot e^{-u^{2}\left(x_{1}-x_{2}\right)^{2}} \int_{-L_{y} / 2}^{+L_{y} / 2} d y_{1} \int_{-L_{y} / 2}^{+L_{y} / 2} d y_{2} \\
& \cdot e^{-u^{2}\left(y_{1}-y_{2}\right)^{2}} .
\end{aligned}
$$

One can write the expression in (5) as

$$
\begin{aligned}
U\left(L_{x}, L_{y}\right)= & \frac{k_{e} \sigma^{2}}{\sqrt{\pi}} \int_{0}^{\infty} d u \int_{-L_{x} / 2}^{+L_{x} / 2} d x_{2} f\left(u, x_{2}, L_{x}\right) \\
& \cdot \int_{-L_{y} / 2}^{+L_{y} / 2} d y_{2} f\left(u, y_{2}, L_{y}\right),
\end{aligned}
$$


where the two functions, $f\left(u, x_{2}, L_{x}\right)$ and $f\left(u, y_{2}, L_{y}\right)$, are defined and calculated in Appendix A. Two of the resulting integrals in (6) are calculated in Appendix B. With help from (B.8) and (B.9) and after simple manipulations one rewrites the expression in (6) as

$$
U\left(L_{x}, L_{y}\right)=\frac{k_{e} Q^{2}}{\sqrt{\pi}} \int_{0}^{\infty} d u g\left(u L_{x}\right) g\left(u L_{y}\right),
$$

where the auxiliary function, $g(t)$, has the following form [see (B.7)]:

$$
g(t)=\sqrt{\pi} \frac{\operatorname{erf}(t)}{t}+\frac{e^{-t^{2}}-1}{t^{2}} .
$$

It can be easily verified from (7) that $U\left(L_{x}, L_{y}\right)$ diverges if $L_{x}=0\left(\right.$ or $\left.L_{y}=0\right)$ or both $L_{x}=L_{y}=0$. Note that $g(t=0)=$ 1 and $\int_{0}^{\infty} d t g(t)=\infty$ for such a situation.

By using the expression for $g(t)$ given in (8) one can write the quantity in (7) more explicitly as

$$
\begin{aligned}
& U\left(L_{x}, L_{y}\right)=\frac{k_{e} Q^{2}}{\sqrt{\pi}} \int_{0}^{\infty} d u \\
& \quad\left\{\frac{\pi}{L_{x} L_{y}} \frac{\operatorname{erf}\left(u L_{x}\right) \operatorname{erf}\left(u L_{y}\right)}{u^{2}}\right. \\
& +\frac{\sqrt{\pi}}{L_{x} L_{y}^{2}} \frac{\operatorname{erf}\left(u L_{x}\right)}{u^{3}}\left[e^{-\left(u L_{y}\right)^{2}}-1\right] \\
& +\frac{\sqrt{\pi}}{L_{x}^{2} L_{y}} \frac{\operatorname{erf}\left(u L_{y}\right)}{u^{3}}\left[e^{-\left(u L_{x}\right)^{2}}-1\right] \\
& \left.+\frac{1}{L_{x}^{2} L_{y}^{2}} \frac{1}{u^{4}}\left[e^{-\left(u L_{x}\right)^{2}}-1\right]\left[e^{-\left(u L_{y}\right)^{2}}-1\right]\right\} .
\end{aligned}
$$

Calculation of the quantity in (9) involves three different types of integrals as listed below:

$$
\begin{aligned}
& \int_{0}^{\infty} d x \frac{\operatorname{erf}(a x) \operatorname{erf}(b x)}{x^{2}} \\
& =\frac{2}{\sqrt{\pi}}\left[a \sinh ^{-1}\left(\frac{b}{\sqrt{a^{2}}}\right)+b \sinh ^{-1}\left(\frac{a}{\sqrt{b^{2}}}\right)\right], \\
& \int_{0}^{\infty} \frac{d x}{x^{3}} \operatorname{erf}(a x)\left(e^{-b^{2} x^{2}}-1\right) \\
& =a\left(\sqrt{a^{2}}-\sqrt{a^{2}+b^{2}}\right)-b^{2} \sinh ^{-1}\left(\frac{a}{\sqrt{b^{2}}}\right) \\
& \int_{0}^{\infty} \frac{d x}{x^{4}}\left(e^{-a^{2} x^{2}}-1\right)\left(e^{-b^{2} x^{2}}-1\right) \\
& =\frac{2}{3} \sqrt{\pi}\left[\left(a^{2}+b^{2}\right)^{3 / 2}-\left(a^{2}\right)^{3 / 2}-\left(b^{2}\right)^{3 / 2}\right],
\end{aligned}
$$

where $a, b$ are real constants and $\sinh ^{-1}(x)=\ln \left(x+\sqrt{x^{2}+1}\right)$ is the inverse sine hyperbolic function. At this juncture, we point out that the integrals shown in (10) are not ordinary table integrals. Thus, for the benefit of the reader, all the details of the calculations of such integrals are provided in Appendices C, D, and E, respectively. The key point made is that all the one-dimensional integrals appearing in (9) can be calculated exactly. After some careful algebraic manipulations we were able to write the final result for the Coulomb selfenergy of a uniformly charged rectangular plate as

$$
\begin{aligned}
& U\left(L_{x}, L_{y}\right)=k_{e} Q^{2}\left\{\frac{1}{L_{x}} \sinh ^{-1}\left(\frac{L_{x}}{L_{y}}\right)\right. \\
& +\frac{1}{L_{y}} \sinh ^{-1}\left(\frac{L_{y}}{L_{x}}\right) \\
& \left.+\frac{1}{3}\left[\frac{L_{x}}{L_{y}^{2}}+\frac{L_{y}}{L_{x}^{2}}-\left(\frac{1}{L_{x}^{2}}+\frac{1}{L_{y}^{2}}\right) \sqrt{L_{x}^{2}+L_{y}^{2}}\right]\right\} .
\end{aligned}
$$

Note that the expression in (11) allows one to immediately see the expected result:

$$
U\left(L_{x}, L_{y}\right)=U\left(L_{y}, L_{x}\right) .
$$

The expression in (11) can be cast in a different form to show how the energy varies with size. In such a scenario, one must assume that at least one of the lengths $\left(L_{x}\right.$ or $\left.L_{y}\right)$ is nonzero. For instance, let us assume that $L_{x} \neq 0$ which means that we can use $k_{e} Q^{2} / L_{x}$ as unit of energy. Let us now introduce a dimensionless parameter:

$$
\alpha=\frac{L_{y}}{L_{x}} \geq 0 .
$$

For such a choice one has

$$
U\left(L_{x}, L_{y}=\alpha L_{x}\right)=\frac{k_{e} Q^{2}}{L_{x}} F(\alpha),
$$

where

$$
\begin{aligned}
F(\alpha)= & \sinh ^{-1}\left(\frac{1}{\alpha}\right)+\frac{1}{\alpha} \sinh ^{-1}(\alpha) \\
& +\frac{1}{3}\left[\frac{1}{\alpha^{2}}+\alpha-\left(1+\frac{1}{\alpha^{2}}\right) \sqrt{1+\alpha^{2}}\right] .
\end{aligned}
$$

A plot of $F(\alpha)$ as a function of $\alpha$ is shown in Figure 2. Note that $F(\alpha \rightarrow 0) \rightarrow \infty$ which is consistent with the well-known fact that the Coulomb self-energy of a uniformly charged line diverges if charges interact with a standard (inverse distance) Coulomb interaction potential.

For the special case of a uniformly charged square plate we have

$$
L_{x}=L_{y}=L .
$$

The expression for the Coulomb self-energy of a uniformly charged square plate becomes

$$
\begin{aligned}
U(L, L) & =\frac{k_{e} Q^{2}}{L}\left[2 \sinh ^{-1}(1)+\frac{2(1-\sqrt{2})}{3}\right] \\
& =\frac{k_{e} Q^{2}}{L}\left[2 \ln (1+\sqrt{2})+\frac{2(1-\sqrt{2})}{3}\right],
\end{aligned}
$$




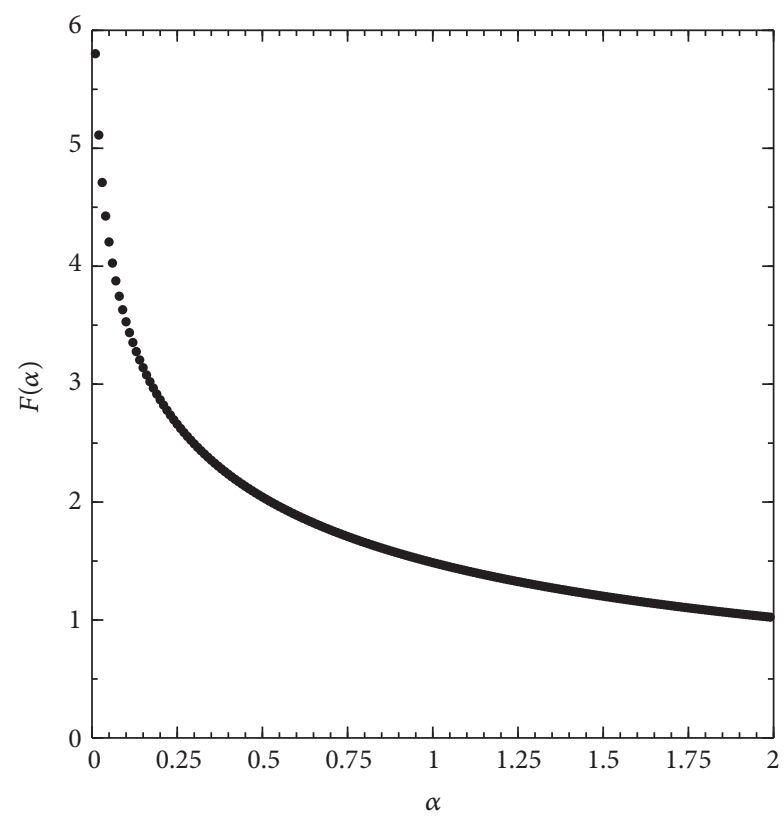

FIgURE 2: The Coulomb self-energy of a uniformly charged rectangular plate, $U\left(L_{x}, L_{y}\right)$, can be expressed in units of $k_{e} Q^{2} / L_{x}$ for $L_{x} \neq 0$ in terms of a function, $F(\alpha)$, where $\alpha=L_{y} / L_{x} \geq 0$ is a nonnegative dimensionless parameter. The value $\alpha=1$ represents a uniformly charged square. The value $\alpha=0$ represents a uniformly charged line with length, $L_{x}$, in which a total charge, $Q$, is uniformly spread over its length. The values of parameter $\alpha$ in the plot vary from $\alpha=0.01$ to $\alpha=2$ in steps of $\Delta \alpha=0.01$.

where we used the fact that $\sinh ^{-1}(1)=\ln (1+\sqrt{2})$. This result can also be proven directly starting from (7) after noting that

$$
U(L, L)=\frac{k_{e} Q^{2}}{L} \frac{1}{\sqrt{\pi}} \int_{0}^{\infty} d t[g(t)]^{2} .
$$

The resulting integral, $\int_{0}^{\infty} d t[g(t)]^{2}$, is calculated in Appendix F. Substitution of the result from (F.6) to (18) leads to the expression in (17) which represents the exact value of the Coulomb self-energy of a uniformly charged square plate [28] with length $L$ containing a total amount of charge $Q$.

\section{Conclusions}

To conclude, we calculated in exact closed form the Coulomb self-energy (namely, the amount of stored electrostatic energy) of a uniformly charged rectangular plate with arbitrary length, $L_{x}$, and width, $L_{y}$. We introduced a suitable mathematical transformation of variables which enabled us to obtain the Coulomb self-energy in one-dimensional integral form as reported in (7). Such an integral expression was used as a starting point to verify that the Coulomb self-energy diverges in the limit when at least one of the lengths goes to zero (namely, $L_{x} \rightarrow 0$ or $L_{y} \rightarrow 0$ limit). From that point on, we focused our attention on the general case scenario of arbitrary length and width. The exact closed form solution of the problem for arbitrary $L_{x}$ and $L_{y}$ was obtained after calculating the tedious one-dimensional integrals appearing in (9).

The exact expression of the Coulomb self-energy of a uniformly charged rectangular plate is written in (11) and depends on length and width (thus, shape) of the plate. The general result for a uniformly charged rectangular plate reduces to the expected value of a square plate when length and width are equal [see (17)]. Knowing the exact form of the Coulomb self-energy of a uniformly charged rectangular plate as a function of length/width and shape parameters may help one analyze how electrostatic energy is stored in various uniformly charged plates with rectangular shapes without resorting to approximations. This can be useful to understand the properties of various electronic nanosystems made up of charged nanoplates. Such situations are often encountered in studies of 2D nanoscale structures or metallic thin films where the dominant interactions are electrostatic. It is also important to remark that several integral formulae that were derived in the process do not appear to be standard and, thus, special care was required when dealing with them. Therefore, some mathematical derivations (typically, those appearing in the Appendices) are deemed of interest on their own from a mathematical point of view.

\section{Appendix}

\section{A. Functions, $f\left(u, x_{2}, L_{x}\right)$ and $f\left(u, y_{2}, L_{y}\right)$}

Let us consider the following function:

$$
f\left(u, x_{2}, L_{x}\right)=\int_{-L_{x} / 2}^{+L_{x} / 2} d x_{1} e^{-u^{2}\left(x_{1}-x_{2}\right)^{2}} .
$$

We denote $v=x_{1}-x_{2}$ and rewrite the expression in (A.1) as

$$
f\left(u, x_{2}, L_{x}\right)=\int_{-L_{x} / 2-x_{2}}^{+L_{x} / 2-x_{2}} d v e^{-u^{2} v^{2}} .
$$

The following integral formula applies:

$$
\int d x e^{-a^{2} x^{2}}=\frac{\sqrt{\pi}}{2 a} \operatorname{erf}(a x),
$$

where $\operatorname{erf}(x)$ is an error function and it is assumed that $a$ is a real constant. Using the fact that $\operatorname{erf}(-x)=-\operatorname{erf}(x)$, it is straightforward to obtain

$$
\begin{aligned}
& f\left(u, x_{2}, L_{x}\right) \\
& =\frac{\sqrt{\pi}}{2 u}\left\{\operatorname{erf}\left[u\left(\frac{L_{x}}{2}+x_{2}\right)\right]+\operatorname{erf}\left[u\left(\frac{L_{x}}{2}-x_{2}\right)\right]\right\} .
\end{aligned}
$$

The calculation of

$$
f\left(u, y_{2}, L_{y}\right)=\int_{-L_{y} / 2}^{+L_{y} / 2} d y_{1} e^{-u^{2}\left(y_{1}-y_{2}\right)^{2}}
$$

follows a similar path. One ends up with the following result:

$$
\begin{aligned}
& f\left(u, y_{2}, L_{y}\right)=\frac{\sqrt{\pi}}{2 u}\left\{\operatorname{erf}\left[u\left(\frac{L_{y}}{2}+y_{2}\right)\right]\right. \\
& \left.+\operatorname{erf}\left[u\left(\frac{L_{y}}{2}-y_{2}\right)\right]\right\} .
\end{aligned}
$$


B. Calculation of $\int_{-L_{x} / 2}^{+L_{x} / 2} d x_{2} f\left(u, x_{2}, L_{x}\right)$ and

$$
\int_{-L_{y} / 2}^{+L_{y} / 2} d y_{2} f\left(u, y_{2}, L_{y}\right)
$$

We want to calculate the integral

$$
\int_{-L_{x} / 2}^{+L_{x} / 2} d x_{2} f\left(u, x_{2}, L_{x}\right),
$$

where

$$
\begin{aligned}
& f\left(u, x_{2}, L_{x}\right) \\
& =\frac{\sqrt{\pi}}{2 u}\left\{\operatorname{erf}\left[u\left(\frac{L_{x}}{2}+x_{2}\right)\right]+\operatorname{erf}\left[u\left(\frac{L_{x}}{2}-x_{2}\right)\right]\right\} .
\end{aligned}
$$

By substituting the expression from (B.2) into the integral expression in (B.1) one obtains

$$
\begin{aligned}
& \int_{-L_{x} / 2}^{+L_{x} / 2} d x_{2} f\left(u, x_{2}, L_{x}\right) \\
& \quad=\frac{\sqrt{\pi}}{2 u}\left\{\int_{-L_{x} / 2}^{+L_{x} / 2} d x_{2} \operatorname{erf}\left[u\left(\frac{L_{x}}{2}+x_{2}\right)\right]\right. \\
& \left.\quad+\int_{-L_{x} / 2}^{+L_{x} / 2} d x_{2} \operatorname{erf}\left[u\left(\frac{L_{x}}{2}-x_{2}\right)\right]\right\} .
\end{aligned}
$$

One simplifies the calculation of the first integral in (B.3) by introducing a dummy variable, $z=u\left(L_{x} / 2+x_{2}\right)$. To simplify the second integral in (B.3), one introduces a dummy variable, $z=u\left(L_{x} / 2-x_{2}\right)$. Simple algebraic transformations lead to the following result:

$$
\int_{-L_{x} / 2}^{+L_{x} / 2} d x_{2} f\left(u, x_{2}, L_{x}\right)=\frac{\sqrt{\pi}}{u^{2}} \int_{0}^{u L_{x}} d z \operatorname{erf}(z)
$$

We now use the following integration formula:

$$
\int d x \operatorname{erf}(x)=x \operatorname{erf}(x)+\frac{1}{\sqrt{\pi}} e^{-x^{2}} .
$$

One obtains

$$
\begin{aligned}
& \int_{-L_{x} / 2}^{+L_{x} / 2} d x_{2} f\left(u, x_{2}, L_{x}\right) \\
& =\frac{\sqrt{\pi}\left(u L_{x}\right) \operatorname{erf}\left(u L_{x}\right)+e^{-\left(u L_{x}\right)^{2}}-1}{u^{2}} .
\end{aligned}
$$

Let us now introduce the following auxiliary function:

$$
\begin{aligned}
g(t) & =\frac{\sqrt{\pi} t \operatorname{erf}(t)+e^{-t^{2}}-1}{t^{2}} \\
& =\sqrt{\pi} \frac{\operatorname{erf}(t)}{t}+\frac{e^{-t^{2}}-1}{t^{2}} .
\end{aligned}
$$

As a result one writes

$$
\int_{-L_{x} / 2}^{+L_{x} / 2} d x_{2} f\left(u, x_{2}, L_{x}\right)=L_{x}^{2} g\left(u L_{x}\right)
$$

where $g(t)$ is defined in (B.7). By using the same approach one has

$$
\int_{-L_{y} / 2}^{+L_{y} / 2} d y_{2} f\left(u, y_{2}, L_{y}\right)=L_{y}^{2} g\left(u L_{y}\right)
$$

\section{Calculation of the Integral $\int_{0}^{\infty}\left(d x / x^{2}\right) \operatorname{erf}(a x) \operatorname{erf}(b x)$}

We consider the following integral:

$$
I(a, b)=\int_{0}^{\infty} d x \frac{\operatorname{erf}(a x) \operatorname{erf}(b x)}{x^{2}},
$$

where $a, b$ are real constants and $\operatorname{erf}(x)$ is an error function. We use integration by parts $\left(\int u d v=u v-\int v d u\right)$, where $u=$ $\operatorname{erf}(a x) \operatorname{erf}(b x)$ and $d v=d x / x^{2}$. Straightforward calculations lead to

$$
\begin{aligned}
& I(a, b)=\frac{2}{\sqrt{\pi}}\left[a \int_{0}^{\infty} d x e^{-a^{2} x^{2}} \frac{\operatorname{erf}(b x)}{x}\right. \\
& \left.+b \int_{0}^{\infty} d x e^{-b^{2} x^{2}} \frac{\operatorname{erf}(a x)}{x}\right] .
\end{aligned}
$$

The result in (C.2) was obtained after noting that

$$
\lim _{x \rightarrow 0} \frac{\operatorname{erf}(a x) \operatorname{erf}(b x)}{x}=\lim _{x \rightarrow \infty} \frac{\operatorname{erf}(a x) \operatorname{erf}(b x)}{x}=0 .
$$

The first integral appearing in the right-hand-side of (C.2) can be calculated from the following result:

$$
\int_{0}^{\infty} d x e^{-a^{2} x^{2}} \frac{\operatorname{erf}(b x)}{x}=\sinh ^{-1}\left(\frac{b}{\sqrt{a^{2}}}\right)
$$

where $\sinh ^{-1}(x)=\ln \left(x+\sqrt{x^{2}+1}\right)$ is the inverse sine hyperbolic function. The formula in (C.4) can be used to calculate the second integral appearing on the right-handside of (C.2) with the result:

$$
\int_{0}^{\infty} d x e^{-b^{2} x^{2}} \frac{\operatorname{erf}(a x)}{x}=\sinh ^{-1}\left(\frac{a}{\sqrt{b^{2}}}\right) .
$$

Hence, we were able to calculate each of the two integrals appearing in the right-hand-side of (C.2). Such integrals are given, respectively, in (C.4) and (C.5). At this juncture, the last step left is to substitute the results from (C.4) and (C.5) into the expression in (C.2) to obtain

$$
\begin{aligned}
& I(a, b) \\
& \quad=\frac{2}{\sqrt{\pi}}\left[a \sinh ^{-1}\left(\frac{b}{\sqrt{a^{2}}}\right)+b \sinh ^{-1}\left(\frac{a}{\sqrt{b^{2}}}\right)\right] .
\end{aligned}
$$

\section{Calculation of the Integral

$$
\int_{0}^{\infty}\left(d x / x^{3}\right) \operatorname{erf}(a x)\left(e^{-b^{2} x^{2}}-1\right)
$$

Let us consider the following integral:

$$
I(a, b)=\int_{0}^{\infty} \frac{d x}{x^{3}} \operatorname{erf}(a x)\left(e^{-b^{2} x^{2}}-1\right),
$$


where $a, b$ are real constants and $\operatorname{erf}(x)$ is an error function. Let us first consider the following simpler integral, $I(a, b=1)$, which can be written as

$$
I(a, b=1)=I_{1}(a)-I_{2}(a),
$$

where

$$
\begin{aligned}
& I_{1}(a)=\int_{0}^{\infty} \frac{d x}{x^{3}} e^{-x^{2}} \operatorname{erf}(a x), \\
& I_{2}(a)=\int_{0}^{\infty} \frac{d x}{x^{3}} \operatorname{erf}(a x) .
\end{aligned}
$$

It has been proven [10] that while both $I_{1}(a)$ and $I_{2}(a)$ have singularities (infinities), all such singularities cancel out when the difference, $I_{1}(a)-I_{2}(a)$, is calculated. After careful integration [10] one obtains

$$
\begin{aligned}
I_{1}(a)= & \left.\frac{1}{2} \frac{e^{-x^{2}} \operatorname{erf}(a x)}{x^{2}}\right|_{x \rightarrow 0}-\sinh ^{-1}(a) \\
& +\left.\frac{a}{\sqrt{\pi}} \frac{e^{-\left(a^{2}+1\right) x^{2}}}{x}\right|_{x \rightarrow 0}-a \sqrt{a^{2}+1}, \\
I_{2}(a)= & -a \sqrt{a^{2}}+\left.\frac{a}{\sqrt{\pi}} \frac{e^{-a^{2} x^{2}}}{x}\right|_{x \rightarrow 0}+\left.\frac{\operatorname{erf}(a x)}{2 x^{2}}\right|_{x \rightarrow 0} .
\end{aligned}
$$

By substituting the results from (D.4) into the expression for $I(a, b=1)$ in (D.2), the following result is obtained:

$$
I(a, b=1)=a\left(\sqrt{a^{2}}-\sqrt{a^{2}+1}\right)-\sinh ^{-1}(a) .
$$

Since we are assuming that both $a, b$ are real constants then $b^{2}=|b|^{2} \geq 0$. The key observation is that the integral in (D.1) can be cast in the form of (D.5) by changing to a new variable, $y=|b| x$. The rest of the calculations are straightforward and the final result is

$$
I(a, b)=a\left(\sqrt{a^{2}}-\sqrt{a^{2}+b^{2}}\right)-b^{2} \sinh ^{-1}\left(\frac{a}{\sqrt{b^{2}}}\right) .
$$

\section{E. Calculation of the Integral}

$$
\int_{0}^{\infty}\left(d x / x^{4}\right)\left(e^{-a^{2} x^{2}}-1\right)\left(e^{-b^{2} x^{2}}-1\right)
$$

We consider the integral

$$
I(a, b)=\int_{0}^{\infty} \frac{d x}{x^{4}}\left(e^{-a^{2} x^{2}}-1\right)\left(e^{-b^{2} x^{2}}-1\right),
$$

where $a, b$ are real constants. The integral in (E.1) can be written as

$$
\begin{aligned}
I(a, b)= & \int_{0}^{\infty} \frac{d x}{x^{4}} e^{-\left(a^{2}+b^{2}\right) x^{2}}-\int_{0}^{\infty} \frac{d x}{x^{4}} e^{-a^{2} x^{2}} \\
& -\int_{0}^{\infty} \frac{d x}{x^{4}} e^{-b^{2} x^{2}}+\int_{0}^{\infty} \frac{d x}{x^{4}} .
\end{aligned}
$$

Each of the integrals in (E.2) can be calculated by using the following result:

$$
\begin{aligned}
\int \frac{d x}{x^{4}} e^{-a^{2} x^{2}}= & e^{-a^{2} x^{2}}\left(-\frac{1}{3 x^{3}}+\frac{2 a^{2}}{3 x}\right) \\
& +\frac{2}{3} a^{3} \sqrt{\pi} \operatorname{erf}(a x),
\end{aligned}
$$

where $\operatorname{erf}(x)$ is an error function. We recall that

$$
\begin{aligned}
& \lim _{x \rightarrow 0} \operatorname{erf}(a x)=0 \\
& \lim _{x \rightarrow \infty} \operatorname{erf}(a x)= \pm 1=\frac{a}{|a|} .
\end{aligned}
$$

As a result the value of the determinate integral in (E.3) (with lower boundary, $x=0$, and upper boundary, $x=\infty$ ) is

$$
\begin{aligned}
\int_{0}^{\infty} \frac{d x}{x^{4}} e^{-a^{2} x^{2}}= & \left.e^{-a^{2} x^{2}}\left(\frac{1}{3 x^{3}}-\frac{2 a^{2}}{3 x}\right)\right|_{x \rightarrow 0} \\
& +\frac{2}{3} \sqrt{\pi}\left(a^{2}\right)^{3 / 2}
\end{aligned}
$$

We wrote $|a|^{3}=\left(a^{2}\right)^{3 / 2}$ in order to keep the result in (E.5) as general as possible. This way one can easily use (E.5) when " $a$ " is formally replaced by " $\left(a^{2}+b^{2}\right)$." The divergent term (in the $x \rightarrow 0$ limit) in the right-hand-side of (E.5) requires special attention. Each of the four integrals appearing in (E.2) contains a divergent term (in the $x \rightarrow 0$ limit). However, one can prove that all such divergent terms cancel out exactly when added up in the right-hand-side expression of (E.2). The final result is

$$
I(a, b)=\frac{2}{3} \sqrt{\pi}\left[\left(a^{2}+b^{2}\right)^{3 / 2}-\left(a^{2}\right)^{3 / 2}-\left(b^{2}\right)^{3 / 2}\right] .
$$

\section{F. Calculation of $\int_{0}^{\infty} d t[g(t)]^{2}$}

We want to calculate the following integral:

$$
\int_{0}^{\infty} d t[g(t)]^{2}
$$

where

$$
g(t)=\sqrt{\pi} \frac{\operatorname{erf}(t)}{t}+\frac{e^{-t^{2}}-1}{t^{2}} .
$$

One can write the quantity in (F.1) as

$$
\begin{aligned}
\int_{0}^{\infty} d t[g(t)]^{2}= & \pi \int_{0}^{\infty} d t\left[\frac{\operatorname{erf}(t)}{t}\right]^{2} \\
& +2 \sqrt{\pi} \int_{0}^{\infty} \frac{d t}{t^{3}} \operatorname{erf}(t)\left(e^{-t^{2}}-1\right) \\
& +\int_{0}^{\infty} \frac{d t}{t^{4}}\left(e^{-t^{2}}-1\right)^{2}
\end{aligned}
$$


All the integrals appearing above can be calculated exactly. We list the results below:

$$
\int_{0}^{\infty} d t\left[\frac{\operatorname{erf}(t)}{t}\right]^{2}=\frac{4}{\sqrt{\pi}} \sinh ^{-1}(1)
$$

where $\sinh ^{-1}(x)=\ln \left(x+\sqrt{x^{2}+1}\right)$ is the inverse sine hyperbolic function

$$
\begin{gathered}
\int_{0}^{\infty} \frac{d t}{t^{3}} \operatorname{erf}(t)\left(e^{-t^{2}}-1\right)=1-\sqrt{2}-\sinh ^{-1}(1), \\
\int_{0}^{\infty} \frac{d t}{t^{4}}\left(e^{-t^{2}}-1\right)^{2}=\frac{4 \sqrt{\pi}}{3}(\sqrt{2}-1) .
\end{gathered}
$$

By putting all together one has

$$
\int_{0}^{\infty} d t[g(t)]^{2}=2 \sqrt{\pi}\left[\sinh ^{-1}(1)+\frac{1-\sqrt{2}}{3}\right] .
$$

\section{Competing Interests}

The author declares no competing interests.

\section{Acknowledgments}

This research was supported in part by US Army Research Office (ARO) Grant no. W911NF-13-1-0139 and National Science Foundation (NSF) Grant no. DMR-1410350.

\section{References}

[1] S. Bhowmick and K. Alam, "Dielectric scaling of a top gate silicon nanowire on insulator transistor," Journal of Applied Physics, vol. 104, no. 12, Article ID 124308, 2008.

[2] Y. L. Bunimovich, Y. S. Shin, W.-S. Yeo, M. Amori, G. Kwong, and J. R. Heath, "Quantitative real-time measurements of DNA hybridization with alkylated nonoxidized silicon nanowires in electrolyte solution," Journal of the American Chemical Society, vol. 128, no. 50, pp. 16323-16331, 2006.

[3] T. Cohen-Karni, B. P. Timko, L. E. Weiss, and C. M. Lieber, "Flexible electrical recording from cells using nanowire transistor arrays," Proceedings of the National Academy of Sciences of the United States of America, vol. 106, no. 18, pp. 7309-7313, 2009.

[4] Y. Cui, Q. Q. Wei, H. K. Park, and C. M. Lieber, "Nanowire nanosensors for highly sensitive and selective detection of biological and chemical species," Science, vol. 293, no. 5533, pp. 1289-1292, 2001.

[5] N. Elfström, R. Juhasz, I. Sychugov, T. Engfeldt, A. E. Karlström, and J. Linnros, "Surface charge sensitivity of silicon nanowires: size dependence," Nano Letters, vol. 7, no. 9, pp. 2608-2612, 2007.

[6] O. H. Elibol, B. Reddy, and R. Bashir, "Nanoscale thickness double-gated field effect silicon sensors for sensitive $\mathrm{pH}$ detection in fluid," Applied Physics Letters, vol. 92, no. 19, Article ID 193904, 2008.

[7] X. P. A. Gao, G. F. Zheng, and C. M. Lieber, "Subthreshold regime has the optimal sensitivity for nanowire FET biosensors," Nano Letters, vol. 10, no. 2, pp. 547-552, 2010.
[8] J.-I. Hahm and C. M. Lieber, "Direct ultrasensitive electrical detection of DNA and DNA sequence variations using nanowire nanosensors," Nano Letters, vol. 4, no. 1, pp. 51-54, 2004.

[9] D. A. Walker, B. Kowalczyk, M. O. De La Cruz, and B. A. Grzybowski, "Electrostatics at the nanoscale," Nanoscale, vol. 3, no. 4, pp. 1316-1344, 2011.

[10] O. Ciftja, "Quantitative analysis of shape-sensitive interaction of a charged nanoplate and a charged nanowire," Nano, vol. 10, no. 8, Article ID 1550114, 2015.

[11] S.-L. Jang and S.-S. Liu, "A novel approach for modeling accumulation-mode SOI MOSFETs," Solid-State Electronics, vol. 43, no. 1, pp. 87-96, 1999.

[12] D. A. Muller, T. Sorsch, S. Moccio, F. H. Baumann, K. EvansLutterodt, and G. Timp, "The electronic structure at the atomic scale of ultrathin gate oxides," Nature, vol. 399, no. 6738, pp. 758-761, 1999.

[13] O. Knopfmacher, A. Tarasov, W. Fu et al., "Nernst limit in dualgated Si-nanowire FET sensors," Nano Letters, vol. 10, no. 6, pp. 2268-2274, 2010.

[14] X. X. Zhu, D. Gu, Q. L. Li et al., "Silicon nanowire NVM with high-k gate dielectric stack," Microelectronic Engineering, vol. 86, no. 7-9, pp. 1957-1960, 2009.

[15] J. Robertson, "High dielectric constant oxides," The European Physical Journal Applied Physics, vol. 28, no. 3, pp. 265-291, 2004.

[16] D. Choi, D. Wang, I.-T. Bae et al., " $\mathrm{LiMnPO}_{4}$ nanoplate grown via solid-state reaction in molten hydrocarbon for $\mathrm{Li}$-ion battery cathode," Nano Letters, vol. 10, no. 8, pp. 2799-2805, 2010.

[17] O. Ciftja, "Classical behavior of few-electron parabolic quantum dots," Physica B: Condensed Matter, vol. 404, no. 12-13, pp. 16291631, 2009.

[18] M. B. Tavernier, E. Anisimovas, F. M. Peeters, B. Szafran, J. Adamowski, and S. Bednarek, "Four-electron quantum dot in a magnetic field," Physical Review B, vol. 68, no. 20, Article ID 205305, 2003.

[19] O. Ciftja and M. G. Faruk, "Two-dimensional quantum-dot helium in a magnetic field: variational theory," Physical Review $B$, vol. 72, no. 20, Article ID 205334, 2005.

[20] D. Pfannkuche, R. R. Gerhardts, P. A. Maksym, and V. Gudmundsson, "Theory of quantum dot helium," Physica B: Physics of Condensed Matter, vol. 189, no. 1-4, pp. 6-15, 1993.

[21] J. Kainz, S. A. Mikhailov, A. Wensauer, and U. Rössler, "Quantum dots in high magnetic fields: calculation of groundstate properties," Physical Review B-Condensed Matter and Materials Physics, vol. 65, no. 11, Article ID 115305, 2002.

[22] U. Merkt, J. Huser, and M. Wagner, "Energy spectra of two electrons in a harmonic quantum dot," Physical Review B, vol. 43, no. 9, pp. 7320-7323, 1991.

[23] D. Ceperley, "Ground state of the fermion one-component plasma: a Monte Carlo study in two and three dimensions," Physical Review B, vol. 18, no. 7, pp. 3126-3138, 1978.

[24] B. Bernu, F. Delyon, M. Holzmann, and L. Baguet, "HartreeFock phase diagram of the two-dimensional electron gas," Physical Review B-Condensed Matter and Materials Physics, vol. 84, no. 11, Article ID 115115, 2011.

[25] B. Bernu, F. Delyon, M. Duneau, and M. Holzmann, "Metalinsulator transition in the Hartree-Fock phase diagram of the fully polarized homogeneous electron gas in two dimensions," Physical Review B, vol. 78, no. 24, Article ID 245110, 2008. 
[26] O. Ciftja, B. Sutton, and A. Way, "Energy in a finite twodimensional spinless electron gas," AIP Advances, vol. 3, no. 5, Article ID 052110, 2013.

[27] O. Ciftja, "Hartree-Fock energy of a finite two-dimensional electron gas system in a jellium background," Physica B: Condensed Matter, vol. 458, pp. 92-97, 2015.

[28] O. Ciftja, "Coulomb self-energy and electrostatic potential of a uniformly charged square in two dimensions," Physics Letters A, vol. 374, no. 7, pp. 981-983, 2010. 


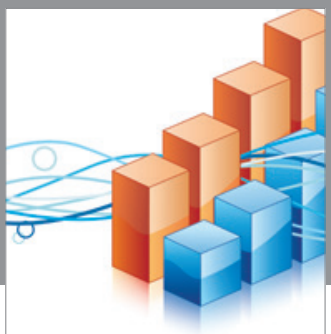

Advances in

Operations Research

vatem alat4

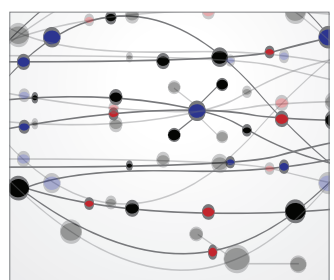

\section{The Scientific} World Journal
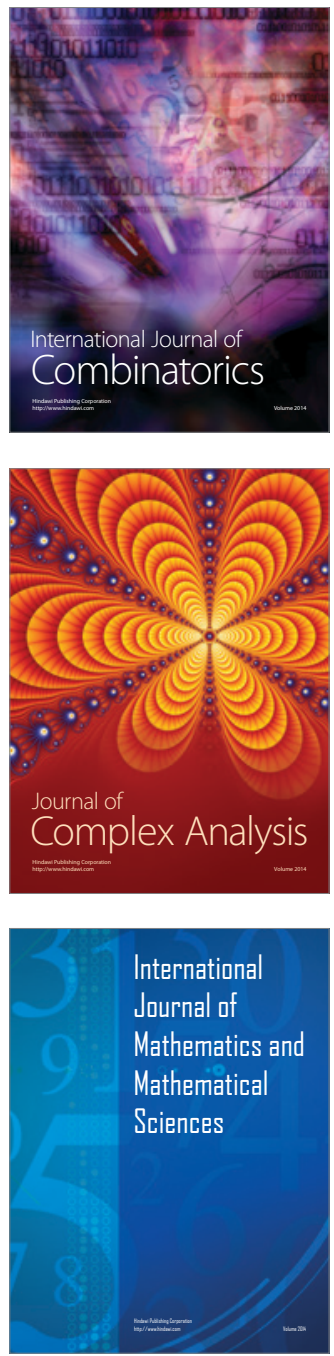
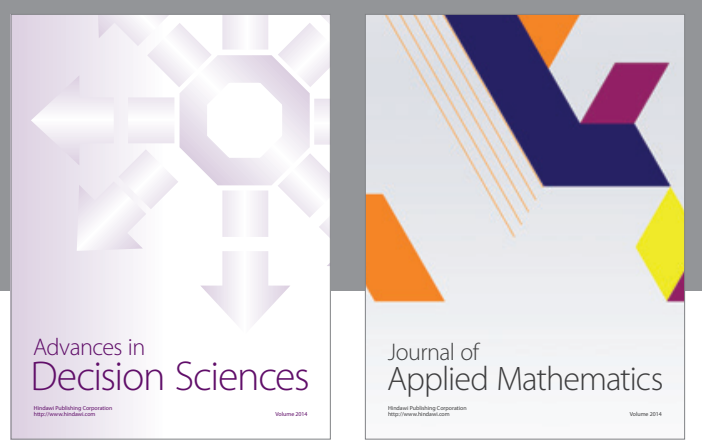

Algebra

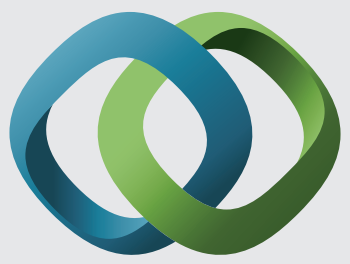

\section{Hindawi}

Submit your manuscripts at

http://www.hindawi.com
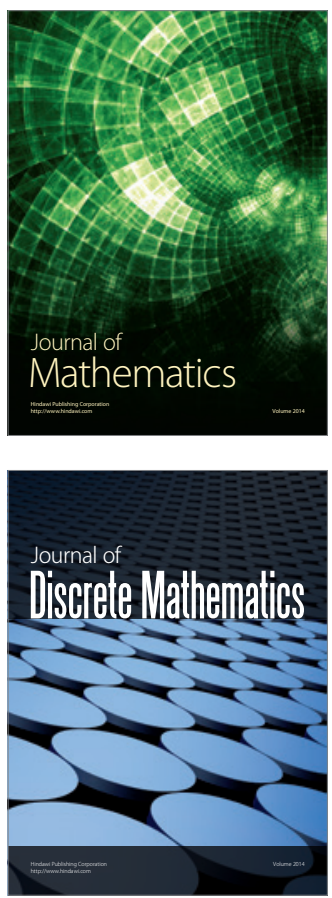

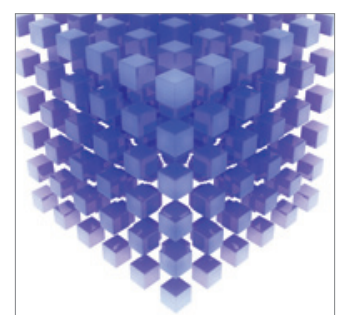

Mathematical Problems in Engineering
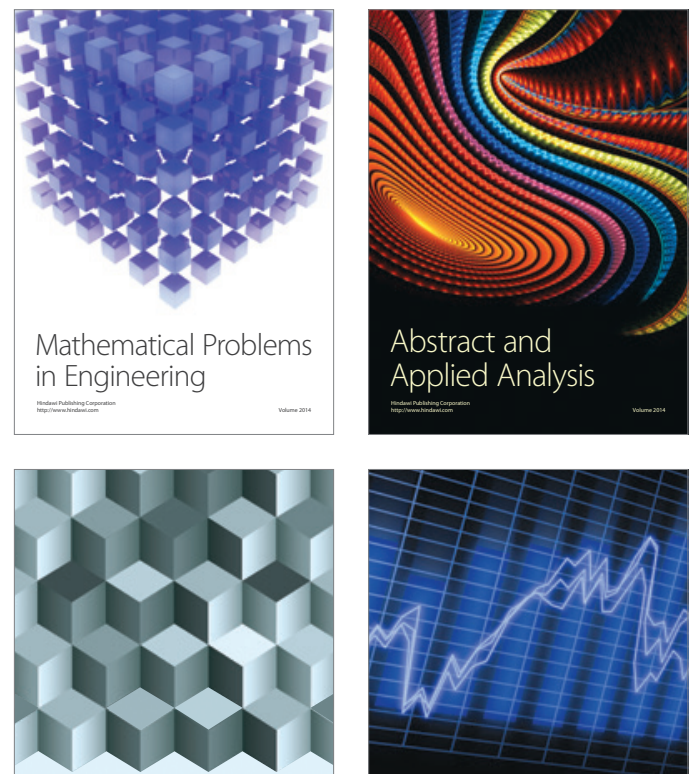

Journal of

Function Spaces

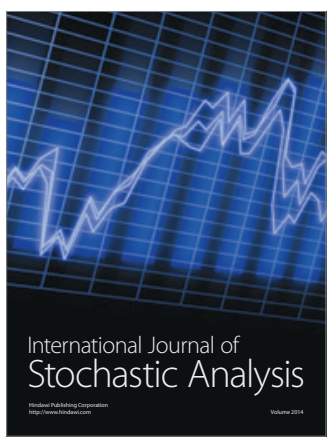

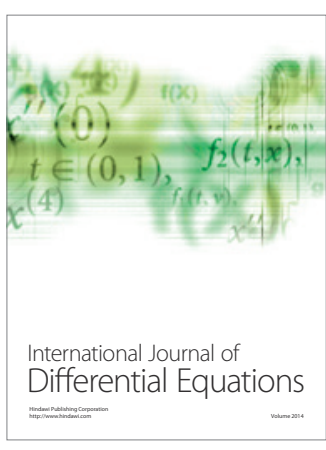
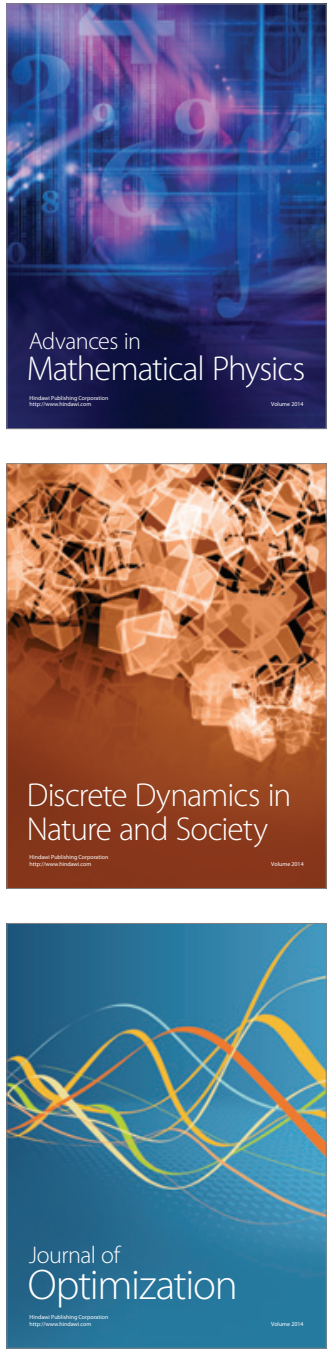